\title{
Cáncer de próstata localizado de alto riesgo tratado mediante prostatectomía radical. Pronóstico y estudio de variables influyentes
}

\author{
Radical prostatectomy for high risk localized prostate cancer. \\ Prognosis and study of influential variables
}

\author{
A. Rincón Mayans ${ }^{2}$, B. Zudaire ${ }^{1}$, J. Brugarolas ${ }^{2}$, J. Rioja ${ }^{2}$, J. Zudaire ${ }^{2}$, D. Rosell ${ }^{2}$, \\ J.E. Robles ${ }^{2}$, J.M. Berian², I. Pascual ${ }^{2}$
}

\section{RESUMEN}

Fundamento. Estudiar la supervivencia libre de progresión bioquímica (SLPB) que ha obtenido un grupo de pacientes de alto riesgo de acuerdo con la clasificación de D’Amico mediante prostatectomía radical.

Identificar las variables clínico-patológicas influyentes en la supervivencia libre de progresión bioquímica y diseñar con ellas, si es posible, un modelo pronóstico.

Material y métodos. Se estudian 232 pacientes, de una serie de 1.054 diagnosticados de cáncer de próstata clínicamente localizado y calificados de alto riesgo en la clasificación de D'Amico (PSA > $20 \mathrm{ng} / \mathrm{ml}$ ó Gleason 8-10 ó T3) tratados mediante prostatectomía radical.

Se estudia la SLPB y se analizan las variables clínico-patológicas recogidas (PSA, Gleason de la biopsia y de la pieza, estadio clínico y patológico, afectación unilateral o bilateral, márgenes de la pieza de prostatectomía, expresión de Ki-67) para identificar si influyen en la SLPB.

Se ha utilizado para el estudio estadístico: tablas de contingencia y para el análisis de la supervivencia: Kaplan-Meyer, Log-rank y modelos de Cox.

Resultados. Estudio descriptivo: PSA: $23,3 \mathrm{ng} / \mathrm{ml}$ (mediana); cGleason 2-6: 33\%; 7: 13\%; 8-10: 54\%; T2: 58\%; Afectación bilateral en la biopsia diagnóstica: 59\%; RNM T2: 60\%; RNM T3: 40\%. pGleason 2-6: 24\%; 7: 28\%; 8-10: 48\%; pT2: 43\%; pT3a: 30\%; pT3b: 27\%; Margen afectado: 51\%; N1:13\%.

Supervivencia libre de progresión: con una media y mediana de seguimiento de 64 meses; el $53 \%$ evidencia progresión bioquímica. La mediana hasta progresión: 42 meses. La supervivencia libre de progresión a 5 y 10 años es $43 \pm 3 \%$ y $26 \pm 7 \%$. El estudio multivariado (modelos de Cox) evidencia que las variables influyentes de forma independiente en la SLPB son la afectación de márgenes (HR: 3,5; 95\% IC.1,9-6,7; p<0001); y Ki67 >10\% (HR: 2,3; 95\% IC: 1,2-4,3; P: 0,009).

Grupos de riesgo: utilizando las dos variables influyentes y utilizando modelos de Cox se diseñan tres grupos de riesgo como mejor modelo: Grupo 1 (0 variables presentes); Grupo 2 (1 variable); Grupo 3 (2 variables). La supervivencia libre de progresión es de $69 \pm 8 \% ; 27 \pm 6 \%$ y $18 \pm 11 \%$ a los 5 años. Las diferencias son significativas entre los tres grupos.

Conclusión. El grupo de alto riesgo de la clasificación de D'Amico es heterogéneo en relación con la progresión bioquímica y puede ser desglosado en tres grupos de riesgo utilizando las dos variables de influencia independiente (márgenes afectados y porcentaje de Ki67).

Palabras clave. Cáncer de próstata. Ki-67. Prostatectomía radical. Alto riesgo. D’Amico. Márgenes quirúrgicos.

\section{ABSTRACT}

Background. To study the biochemical progression-free survival (BPFS) achieved by a group of high risk patients in accordance with D'Amico's classification treated with radical prostatectomy.

To identify the clinical-pathological variables which are influential in biochemical progression-free survival and, if possible, use them to design a prognostic model.

Material and methods. The study involves 232 patients, out of a series of 1,054 , diagnosed with clinically localized prostate cancer, qualified as high risk on D'Amico's classification (PSA $>20 \mathrm{ng} / \mathrm{ml}$ or Gleason score $8-10$ or T3) treated with radical prostatectomy.

The BPFS is studied and the clinical-pathological variables obtained (PSA, Gleason score of the biopsy and of the piece, clinical and pathological study, unilateral or bilateral affectation, margins of the prostatectomy piece, Ki- 67 expression) are analyzed to identify whether they influenced the BPFS.

Contingency tables and tables for survival analysis: Kaplan-Meyer, log-rank and Cox models were used for the statistical study.

Results. Descriptive study: PSA: $23.3 \mathrm{ng} / \mathrm{ml}$ (median); cGleason 2-6: 33\%; 7: $13 \%$; 8-10: $54 \%$; T2: $58 \%$; Bilateral affectation in the diagnostic biopsy 59\%; RNM T2: 60\%; RNM T3: 40\%. pGleason 2-6: 24\%; 7: 28\%; 8-10: 48\%; pT2: 43\%; pT3a: 30\%; pT3b: 27\%; Affected margin: 51\%; N1:13\%.

Progression-free survival: with a mean and median follow-up of 64 months; $53 \%$ show biochemical progression. The median until progression: 42 months. Progression-free survival at 5 and 10 years is $43 \pm 3 \%$ and $26 \pm 7 \%$. The multivariate study (Cox models) shows that the variables that are independently influential in the BPFS are the affectation of margins (HR: 3.5; 95\% IC.1.9-6.7; p<0001); and Ki67 >10\% (HR: 2.3; 95\% IC: $1.2-4.3$; P: 0.009).

Risk groups: using the two influential variables and employing Cox models, three risk groups emerged as the best model: Group 1 ( 0 variables present); Group 2 (1 variable); Group 3 (2 variables). The progression-free survival is $69 \pm 8 \% ; 27 \pm 6 \%$ and $18 \pm 11 \%$ at 5 years. The differences amongst the three groups are significant.

Conclusion. The high risk group according to the D'Amico classification is heterogeneous in relation to biochemical progression and can be broken down into three risk groups using the two independently influential variables (affected margins and Ki67 percentage).

Key words. Prostate cancer Ki-67. Radical prostatectomy. High risk D'Amico. Surgical margin.
1. Departamento de Radiología. Clínica Universidad de Navarra. Pamplona.

2. Departamento de Urología. Clínica Universidad de Navarra. Pamplona.

Recepción: 14 de septiembre de 2011 Aceptación provisional: 25 de octubre de 2011 Aceptación definitiva: 9 de noviembre de 2011

\section{Correspondencia:}

Aníbal Rincón Mayans

Servicio de Urología

Hospital Santiago

C/ Olaguíbel, 29

01004 Vitoria

E-mail: arinconmayans@gmail.com 


\section{INTRODUCCIÓN}

Los pacientes con carcinoma de próstata de alto riesgo constituyen el 15-18\% de los diagnosticados de cáncer de próstata clínicamente localizado ${ }^{1}$. El concepto "alto riesgo" no es unívoco. No hay consenso en la definición y se utilizan o se han utilizado al menos siete clasificaciones diferentes ${ }^{2}$. No es extraño que los resultados sean muy dispares. Nguyen ${ }^{3}$ analizando 4.059 pacientes y utilizando las seis definiciones más utilizadas, evidencia una supervivencia libre de progresión que oscila entre $36-58 \%$ en 5 años y entre $25-43 \%$ en 10 años. El porcentaje de tumores órgano-confinados varía entre $22-62 \%$.

De todos modos, entre todas, la más utilizada ha sido la de D'Amico ${ }^{4}$, a la que implícitamente se la considera la más práctica. Es una clasificación sencilla y sus resultados son comparables a las clasificaciones más sofisticadas ${ }^{2}$. Define tres grupos de riesgo y para ello utiliza el Gleason, el PSA y el estadio clínico. El grupo de alto riesgo incluye a los pacientes con un PSA $>20 \mathrm{ng} /$ ml o un Gleason 8-10 o T3.

Si hay confusión en la definición de los pacientes de alto riesgo, no la hay menos en el tratamiento. El tratamiento óptimo no es claro. El tratamiento hormonal es probablemente la peor opción porque el control local es factor decisivo en la progresión metastásica ${ }^{5}$. En los años 90 el tratamiento más común fue la radioterapia con bloqueo androgénico. Sin embargo, actualmente el tratamiento quirúrgico se abre paso cada vez más como primera opción de tratamiento porque procura un aceptable control local, define la extensión de la enfermedad y determina la necesidad y los posibles tratamientos complementarios.

No obstante la búsqueda de nuevos factores influyentes podría aclarar más el panorama para un tratamiento más racional. Por ello, en este trabajo, hemos analizado las distintas variables clínico-patológicas tradicionalmente estudiadas (PSA, Gleason, márgenes) y valorar, si éstas, se asocian a un peor pronóstico.

También hemos estudiado la expresión de Ki67. En el año $2000^{6}$ pusimos de mani- fiesto la relación de Ki67 con un peor estadio y pronóstico en pacientes operados. Queremos valorar si esa tendencia se confirma en pacientes de alto riesgo.

También mostramos los resultados que la cirugía procura en relación con la supervivencia libre de progresión y si se trata de un grupo homogéneo o pueden definirse dentro de él diferentes grupos de riesgo en relación a las distintas variables estudiadas.

\section{MATERIAL Y MÉTODOS}

Entre 1990 y 2008 hemos realizado 1.054 prostatectomías radicales en nuestro centro a pacientes diagnosticados de adenocarcinoma de próstata clínicamente localizado. De estos, 232 pacientes han sido calificados de alto riesgo de acuerdo con la clasificación de D’Amico (PSA>20 ng/ml ó Gleason 8-10 o estadio T3).

El estudio clínico de todos los pacientes ha consistido en historia clínica, exploración física con tacto rectal, determinación de PSA sérico y biopsia prostática. La estadificación clínica se ha completado hasta el año 2002 con tomografía computarizada y posteriormente con RMN.

Se ha realizado ganmagrafía ósea en los pacientes con PSA superior a $20 \mathrm{ng} / \mathrm{ml}$ y también en pacientes con tumores muy indeferenciados independientemente del valor sérico de PSA.

A todos los pacientes se les ha realizado una prostatectomía radical retropúbica con linfadenectomía ilio-obturatriz. También se ha incluido en el estudio a los pacientes sometidos a prostatectomía radical laparoscópica en este periodo.

Todas las piezas de prostatectomía así como los ganglios linfáticos han sido examinados por el mismo especialista de anatomía patológica utilizando la técnica descrita por True ${ }^{7}$. Para la estadificación se ha utilizado el sistema TNM de la American Joint Committe y la escala de Gleason para clasificar el grado histológico.

Después de la intervención quirúrgica se han realizado determinaciones de PSA a los 3 meses y posteriormente cada 6 me- 
ses. Se ha considerado progresión bioquímica un PSA mayor de $0,4 \mathrm{ng} / \mathrm{ml}^{8}$ en el seguimiento posterior a la intervención.

Ninguno de los pacientes incluidos en el estudio recibió tratamiento (bloqueo hormonal y/o radioterapia externa) antes de evidenciarse progresión bioquímica. Precisamente, para que los resultados no puedan verse afectados por la radioterapia externa y/o bloqueo hormonal que algunos pacientes han recibido posteriormente se ha estudiado la supervivencia libre de progresión bioquímica (SLPB) y no la supervivencia global (supervivencia hasta la muerte) o la supervivencia cáncer específica (supervivencia hasta la muerte por cáncer de próstata) que sí que han podido verse alterados por el tratamiento complementario.

Se han analizado los datos clínicos y patológicos de estos pacientes, incluyendo edad, PSA, estadio clínico, Gleason de la biopsia y de la pieza, estadio ganglionar, extensión extraprostática, invasión de vesiculas seminales, márgenes quirúrgicos afectos y expresión de Ki67.

La determinación de Ki67 se ha realizado en 111 pacientes. Todos los análisis inmunohistoquímicos se han realizado sobre secciones de 4 micrómeros fijadas en formalina y obtenidas del tumor primario incluido en parafina. La inmunohistoquímica se ha realizado con un inmunohistainer automático (Techmate 500; Dako, Copenhague, Dinamarca) con el Envisión + System Dako, en el cual el anticuerpo secundario se acopla a un polímero de dextrano ligado a las moléculas de peroxidasa.

La actividad de peroxidasa endógena se ha neutralizado con peróxido de hidrógeno al 5\%, en solución de metanol durante 30 minutos a temperatura ambiente. La recuperación de antígeno se ha realizado con un tratamiento de microondas durante 20 minutos a 800 watios. Se ha usado suero de conejo para la fase de bloqueo. El anticuerpo primario se ha aplicado durante 120 minutos a temperatura ambiente. Las secciones han sido entonces lavadas con buffer de lavado a temperatura am- biente. El siguiente paso ha sido la adición de reagentes con EnVisión+ System e incubación durante 30 minutos a temperatura ambiente. Las placas han sido lavadas con buffer de lavado y tratadas con una solución de $0,05 \%$ de hidrocloruro de diaminobencina y $0,1 \%$ de peróxido de hidrógeno $0,05 \mathrm{ml} / \mathrm{l}$ de TRIS tampón salino a $\mathrm{pH} 7,4$ a temperatura ambiente, durante 5 minutos. Después del lavado en agua destilada durante 3 minutos las preparaciones han sido teñidas con una solución modificada de hematoxilina de Harris, deshidratada y montada. Para los controles negativos se usa suero normal de cordero en vez de anticuerpo primario (MIB1, Zymed). Se ha expresado el resultado en porcentaje de células teñidas ${ }^{6}$.

Los objetivos del estudio son:

1. Evidenciar la supervivencia libre de progresión bioquímica que la prostatectomía radical obtiene en un grupo de pacientes de alto riesgo de acuerdo con la clasificación de D'Amico.

2. Identificar las variables clínico-patológicas influyentes en dicha supervivencia libre de progresión bioquímica.

3. Diseñar con ellas, si es posible, un modelo pronóstico.

En el estudio estadístico la supervivencia actuarial se ha estudiado mediante la prueba de Kaplan-Meyer. Para la comparación de curvas de supervivencia se ha utilizado la prueba log- Rank. El estudio multivariado de supervivencia y el modelo predictivo se ha realizado utilizando los modelos de regresión de Cox. Cuando ha sido necesario encontrar un punto de corte en variables cuantitativas se ha utilizado el tanteo con curvas ROC. El estudio estadístico se ha realizado con el programa estadístico SPSS versión 15.

\section{RESULTADOS}

El estudio descriptivo de los 232 pacientes se muestra en la tabla 1. 
Tabla 1. Características del grupo de pac de alto riesgo

\begin{tabular}{c|c|c}
\hline \multirow{2}{*}{ Estadio } & Variables clínicas & Variables patológicas \\
& T1c: $41,8 \%(97$ pacientes) & pT2: $43,1 \%$ (100 pacientes) \\
& Resto: $58,2 \%(135$ pacientes) & pT3a: $30,1 \%$ (70 pacientes) \\
pT3b: $26,7 \%$ (62 pacientes)
\end{tabular}

La media y la mediana de PSA del grupo han sido de $24,8 \mathrm{ng} / \mathrm{ml}$ y $23,3 \mathrm{ng} / \mathrm{ml}$; y la media y mediana de Ki67 de $5 \%$ y $9,7 \%$ de células teñidas respectivamente. Cabe destacar que el $43 \%$ ha sido calificado como pT2 y el $51 \%$ ha tenido márgenes afectados.

\section{Estudio de la supervivencia libre de progresión}

La media y mediana de seguimiento del grupo ha sido de 64 meses (5,3 años). El
53\% de los pacientes ha evidenciado progresión bioquímica a lo largo del estudio. La mediana hasta la progresión bioquímica ha sido de 42 meses. La supervivencia libre de progresión bioquímica a 5 y 10 años es de $43 \%$ ( $95 \%$ IC $39 \%-52 \%$ ) y $26 \%$ (95\% IC $18 \%-35 \%)$ respectivamente.

El estudio univariado de los factores clínicos y patológicos que se asocian con la supervivencia libre de progresión se muestra en la tabla 2 (modelos de Cox).

Tabla 2. Estudio univariado de las distintas variables en la supervivencia libre de progresión bioquímica

\begin{tabular}{l|c|c|c}
\hline & HR & $\mathbf{9 5 \%}$ IC HR & p \\
\hline cGleason 2-7 vs 8-10 & 1,03 & $0,7-1,5$ & 0,83 \\
\hline Estadio clínico T1-T2 & 1,5 & $1,06-2,3$ & $\mathbf{0 , 0 2 4}$ \\
\hline Estadio patológico T2-T3 & 2,8 & $1,8-4,3$ & $<\mathbf{0 , 0 0 0 1}$ \\
\hline Ki-67 & 1,03 & $1,01-1,051$ & $<\mathbf{0 , 0 0 0 1}$ \\
\hline Ki-67>10\% & 2,1 & $1,2-3,8$ & $\mathbf{0 , 0 1}$ \\
\hline Biopsia unilateral o bilateral & 1,2 & $0,7-1,9$ & 0,4 \\
\hline Márgenes afectados & 3,4 & $2,2-5,4$ & $<\mathbf{0 , 0 0 0 1}$ \\
\hline Ganglios positivos & & & \\
\hline pGleason 2-7 vs 8-10 & 1,3 & $0,9-2$ & 0,09 \\
\hline PSA & 1,01 & $1,01-1,02$ & $\mathbf{0 , 0 0 1}$ \\
\hline PSA $>20$ ng/ml & 1,5 & $1,05-2,3$ & $\mathbf{0 , 0 2}$ \\
\hline
\end{tabular}


El estudio multivariado de Cox ha puesto de manifiesto que las variables que se asocian de manera independiente con la SLPB es la afectación de márgenes en la pieza de prostatectomía radical (HR: 3,5; 95\% IC: 1,9$6,7 ; \mathrm{p}<0,001)$ y el porcentaje de células que expresan Ki67 (HR: 1,02; 95\% IC 1,01-1,04).

El mejor punto de corte que define la influencia pronóstica de Ki67 ha sido de 10\% de células teñidas (HR: 2,3; 95\% IC: 1,2-4,3; $\mathrm{P}: 0,009)$.

El $75 \%$ de los pacientes con $\mathrm{Ki} 67>10 \%$ han tenido a lo largo del seguimiento fracaso bioquímico (mediana hasta fracaso 5 meses). La supervivencia libre de enfermedad en 5 años ha sido de $24 \%$ (95\% IC: $16-31 \%$ ) frente a $42 \%$ (95\% IC: $34-56 \%$ ) en pacientes con Ki67<10\% (p: 0,01) (Fig. 1).

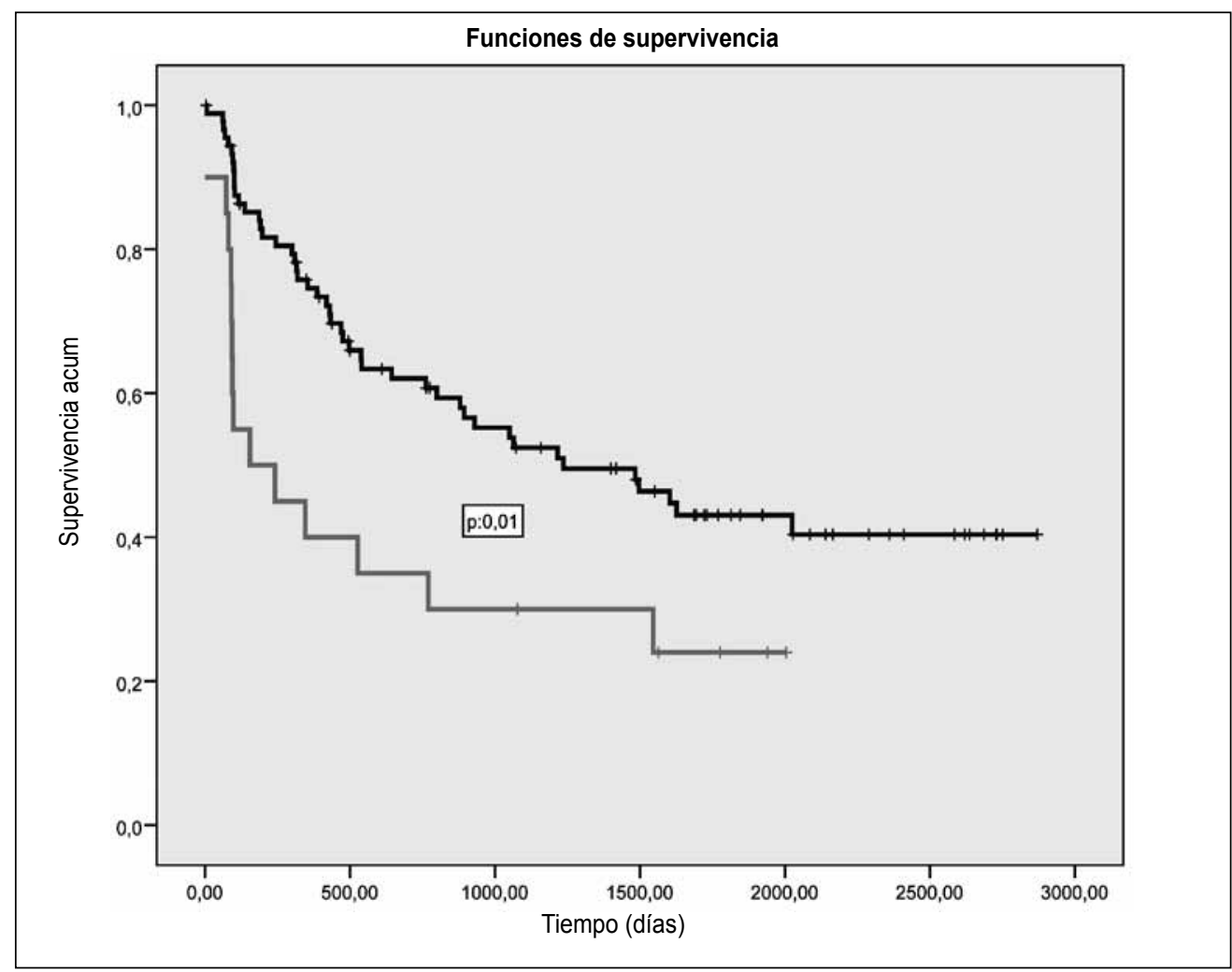

$\mathrm{Ki}<10 \%$ en negro. $\mathrm{Ki}>10 \%$ en gris.

Figura 1. Supervivencia libre de progresión (Ki67<10\% vs >10\%).

El PSA como variable continua está en el margen de la significación (p: 0,09) y no se puede diseñar un modelo dicotómico razonable.

\section{Modelo pronóstico}

Aunque una de las dos variables que se asocian a una peor SLPB se haya determinado en menos del $50 \%$ de la serie estudiada hemos diseñado tres grupos de riesgo:
- Grupo 1: Ki67<10\% y márgenes (-)

- Grupo 2: Ki67 >10\% o márgenes (-)

- Grupo 3: Ki67 >10\% y márgenes (+)

Los tres grupos tenían un pronóstico significativamente diferente: comparados con el primer grupo, el riesgo de progresión bioquímica es 3,5 veces mayor en el grupo 2 y 8,6 veces en el grupo 3 (Tabla 3 . Modelos de Cox). 
Tabla 3. Modelos de Cox. Grupos pronósticos

\begin{tabular}{c|ccccc}
\hline & HR & $\mathbf{9 5 \%}$ IC HR & p & Fracasos & SLPB a 5 años \\
\hline Grupo 1 & 1 & & & $26 \%$ & $69 \pm 8 \%$ \\
\hline Grupo 2 & 3,5 & $1,7-7,3$ & 0,01 & $67 \%$ & $27 \pm 6 \%$ \\
\hline Grupo 3 & 8,6 & $3,4-21,8$ & $<0,0001$ & $82 \%$ & $18 \pm 11 \%$ \\
\hline
\end{tabular}

La supervivencia libre de progresión es a 5 años de $69 \% ; 27 \%$ y $18 \%$ respectivamente (diferencias significativas entre los tres grupos).
En la figura 2 se muestra el estudio de Kaplan-Meyer de los distintos grupos.

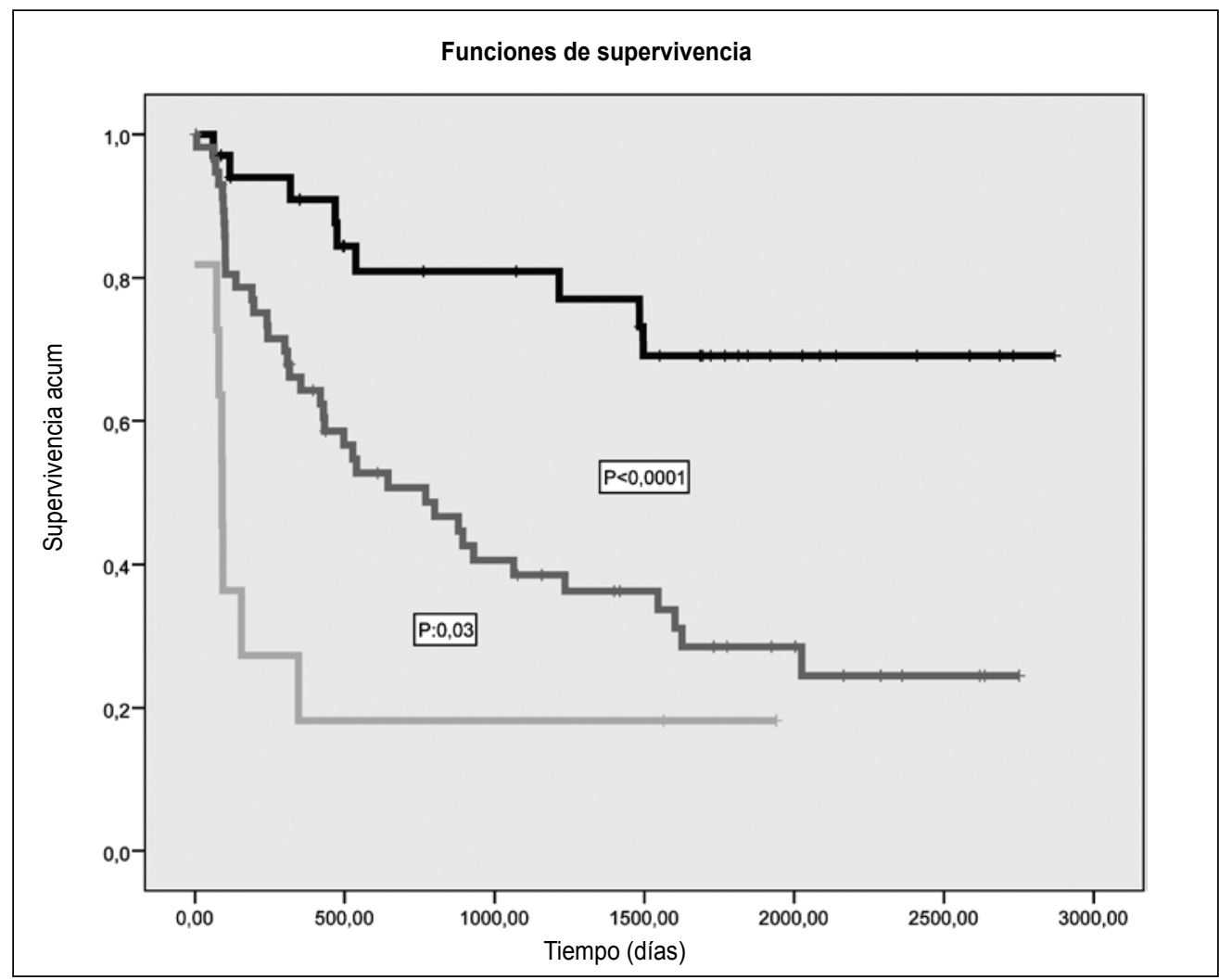

Grupo 1 en negro. Grupo 2 en gris oscuro. Grupo 3 en gris claro.

Figura 2. Supervivencia libre de progresión de los distintos grupos de riesgo

En este trabajo nos hemos basado fundamentalmente en la supervivencia libre de progresión bioquímica ya que ésta no se ha visto modificada o alterada por tratamientos complementarios (bloqueo hormonal y/o radioterapia); ya que ningún paciente del estudio los recibió hasta que se evidenció progresión bioquímica. Sin 
embargo, mostramos los valores obtenidos en supervivencia cáncer específica y global de nuestra serie.

- Supervivencia cáncer específica a 5 y 10 años ha sido de $96 \%$ (95\% IC 94$98 \%$ ) y $89 \%$ (95\% IC 79-96\%).

- Supervivencia global a 5 y 10 años ha sido de $94 \%$ (95\% IC $89-97 \%$ ) y $84 \%$ (95\% IC 76-92\%).

\section{DISCUSIÓN}

En la primera época post-PSA la tendencia editorial era favorable al tratamiento con radioterapia y bloqueo androgénico a los pacientes con adenocarcinoma de próstata de alto riesgo ${ }^{9}$. Entre 1995 y 2001 el porcentaje de $\mathrm{T} 3$ tratados con cirugía radical descendió en EEUU del $18 \%$ al $9 \%{ }^{10}$. Hoy en día la cirugía ha ganado mucho terreno como primera opción debido a la publicación de estudios extensos con buenos resultados en control local, supervivencia específica y supervivencia global ${ }^{9,11}$ y además, aunque no hay estudios aleatorizados que demuestren la superioridad de la cirugía sobre la radioterapia, en estudios de cohortes se ha demostrado en algunos casos la superioridad de la cirugía. Zelefs$\mathrm{ky}^{12}$ comparando pacientes de alto riesgo radiados frente a operados evidencia un riesgo 6,3 veces mayor de tener metástasis en radiados y una diferencia del $8 \%$ en la supervivencia en 8 años. Albertsen ${ }^{13}$ examinando 528 pacientes de alto riesgo (Clasificación de D’Amico) comunica a 10 años una supervivencia específica con cirugía del $90 \%$ frente al $80 \%$ con radioterapia. Tewari ${ }^{14}$ demuestra que el riesgo de progresión bioquímica en operados es $48 \%$ menor que en radiados. Meng y col $^{15}$ indican que los pacientes que eran tratados en primera instancia con radioterapia recibían 3,5 veces más tratamiento hormonal que los que eran sometidos a tratamiento quirúrgico.

La utilización de la cirugía como tratamiento de primera línea se fundamenta en tres razones: el aceptable control local que procura, en la correcta estadificación que informa con claridad del pronóstico y que es imprescindible para valorar la necesidad o no de terapia adyuvante (40-60\% de los pacientes son órgano-confinados; hecho trascendente en la actitud postquirúrgica) y para el diagnóstico precoz de progresión bioquímica si se produce ${ }^{16}$. En la serie de la Clínica Mayo sobre 1.513 pacientes de alto riesgo según clasificación de D'Amico, el $60 \%$ era pT2 (organo-confinado) y con una mediana de seguimiento de 7,7 años sólo el $25 \%$ de los pacientes recibe tratamiento con bloqueo androgénico y menos del $10 \%$ tratamiento complementario con radioterapia. Además el 31\% de los pacientes con gleason clínico mayor o igual a 8 presenta en la pieza de prostatectomía un gleason igual o menor de 7. En nuestro caso el $43 \%$ es pT2 y el $49 \%$ tiene márgenes libres de afectación. Estos pacientes serían sobretratados con radioterapia y bloqueo androgénico.

Hay al menos 8 estudios publicados ${ }^{16}$ con más de 5.000 pacientes en total. A los 10 años la supervivencia libre de progresión es de 35-68\%; la supervivencia libre de metástasis de $32-89 \%$ y la supervivencia específica de $57-92 \%$. No obstante estas cifras deben matizarse. Los resultados son mejores dependiendo del tiempo en que se realiza la cirugía.

Nguyen $^{3}$, en un trabajo con 4.059 pacientes analizados indica que la progresión bioquímica es significativamente menor en los pacientes operados después de 1996. Este hecho es evidente si se comparan las fechas de publicación. Los resultados mejoran en los trabajos más recientes. La razón es la evolución de la clasificación a lo largo del tiempo. Antaño se clasificaban los pacientes de alto riesgo esencialmente sobre la base de ser T3, mientras que las definiciones posteriores incorporan estadio, grado y PSA y la proporción de T3 es mucho menor ${ }^{17}$.

Los dos estudios más extensos corresponden a la Clínica Mayo y a la publicación de los casos reunidos de Baylor College; MSKCC; Cleveland Clinic y Universidad de Michigan[ ${ }^{18}$ ] en ambos casos como nosotros utilizan la clasificación de D'Amico por lo que nuestros datos podrían compararse con los de ellos. En el primer caso ${ }^{9}$ Boorjian analiza 1.513 pacientes de alto 
riesgo (Clasificación de D’Amico) tratados con prostatectomía radical. Aunque el riesgo de muerte es 11,5 veces mayor que en el grupo de bajo riesgo, la supervivencia libre de progresión, supervivencia libre de metástasis y la supervivencia específica a 10 años era de 55\%, 89\% y 95\% respectivamente. En el segundo caso Stephenson ${ }^{18}$ con 1.962 pacientes de alto riesgo (clasificación de D'Amico) muestra una mortalidad por cáncer a 10 y 15 años de $8 \%$ y 19\% respectivamente. Nuestros resultados pueden compararse a los de los dos estudios mencionados en supervivencia específica y global a 10 años (89\% y 84\%).

El estudio más extenso publicado por Nguyen y $\mathrm{col}^{3}$, en nuestra opinión muy realista, sobre 4.059 pacientes de alto riesgo los porcentajes de supervivencia libre de progresión eran de $36-58 \%$ y $25-43 \%$ a 5 y 10 años; valores muy similares a los obtenidos en nuestra serie. Las diferencias con la Clínica $\mathrm{Mayo}^{9}$, con una supervivencia libre de progresión de $55 \%$, podrían deberse a que en nuestro caso solo iniciamos tratamiento hormonal cuando se evidencia progresión bioquímica y en el trabajo mencionado un $24 \%$ de los pacientes recibe bloqueo androgénico adyuvante y un $8 \%$ radioterapia externa después de la cirugía.

En nuestro estudio los factores de influencia pronóstica independiente en la supervivencia libre de progresión son la presencia de márgenes y la expresión celular de Ki67.

El 51\% de nuestros pacientes de alto riesgo tiene márgenes positivos tras el análisis anatomo-patológico de la pieza. Esta cifra, que es elevada, es previsible y se debe a las características clínico-patológicas de estos pacientes. El PSA pre-operatorio y un Gleason elevado se asocia a una mayor presencia de márgenes positivo ${ }^{19}$ y se ha relacionado en otros trabajos como factor de influencia independiente en la supervivencia libre de progresión ${ }^{20}$ en los pacientes de alto riesgo. El PSA en el estudio multivariado no es factor de mal pronóstico al igual que en otros estudios $^{21}$, ni tampoco el estadio clínico o el Gleason de la biopsia.

La originalidad de nuestros resultados radica en la evidencia de que el otro factor influyente en el estudio multivariado es el porcentaje de células que expresan Ki67. Es un antígeno nuclear presente en las fases G1, S, G2 y M del ciclo celular. Se correlaciona con la proliferación celular y su expresión indica de manera indirecta una intensa actividad biológica del tumor y mal pronóstico $^{6,22}$. En un trabajo previo realizado en nuestro centro ${ }^{6}$ ya relacionábamos este antígeno como factor de influencia directa e independiente en la supervivencia libre de progresión y su relación directa con el estadio patológico. La media de expresión en nuestro caso $(9,7 \%)$ es superior a otros estudios ${ }^{6,23}$, pero se debe a que analizamos pacientes de alto riesgo (en el global de nuestra serie la media es de $8,3 \%)$. El mejor punto de corte en los pacientes de alto riesgo es en nuestro estudio el $10 \%$ de células que expresan Ki67, siendo variable de influencia independiente (OR: 2,3; 95\% IC: 1,2-4,3; P: 0,009). Los pacientes con un porcentaje $>10 \%$ progresan en el $75 \%$ a lo largo del estudio (mediana a progresión 5 meses) y la supervivencia libre de progresión es en 5 años de $24 \%$.

Esta variable, la experiencia de Ki67, tiene una notable importancia porque de confirmarse estos resultados en otros estudios estamos frente a un nuevo aporte a la valoración del pronóstico en este tipo de tumores. Además tiene la gran ventaja de que es técnicamente fácil de realizar por inmunohistoquímica en cualquier tejido prostático (material de $\mathrm{RTU}^{24}$, material de biopsia prostática ${ }^{22}$, material tras prostatectomía radical $^{6}$ ) y en cualquier departamento de histopatología.

Utilizando estas dos variables (márgenes quirúrgicos y Ki67) se han diseñado tres grupos de riesgo con diferencias significativas entre ellos, siendo el grupo con ambos factores el que con mayor frecuencia ha requerido tratamiento de segunda línea.

En conclusión, el grupo de alto riesgo de la clasificación de D’Amico es un grupo de mal pronóstico que tradicionalmente ha sido tratado mediante radioterapia $\mathrm{y}$ bloqueo androgénico. Sin embargo, nuevas publicaciones al respecto abogan por el tratamiento quirúrgico por diversas razones: 
reducción en la necesidad de tratamiento de segunda línea, mejor control local de la enfermedad y probablemente mejoría en la supervivencia libre de progresión bioquímica (aunque en este caso debemos ser prudentes a la espera de trabajos aleatorizados prospectivos de largo seguimiento).

En nuestro caso los valores obtenidos en SLPB han sido de $43 \%$ y $26 \%$ a 5 y 10 años mediante prostatectomía radical.

El estudio de las distintas variables clínico-patológicas ha reflejado, en nuestra serie, que la afectación de márgenes quirúrgicos y la expresión de Ki67 son factores pronóstico de influencia independiente.

La afectación del margen quirúrgico es un factor ampliamente conocido por todos como factor pronóstico negativo, sin embargo el Ki-67, menos conocido, es un factor pronóstico influyente en la supervivencia libre de progresión en cáncer de próstata y también como reflejamos en este trabajo en el grupo de alto riesgo.

Y por último, que utilizando estas dos variables (márgenes quirúrgicos y Ki67) se han diseñado tres grupos de riesgo con diferencias significativas entre ellos, siendo el grupo con ambos factores el que peor valores de supervivencia ha obtenido.

\section{BIBLIOGRAFÍA}

1. Cooperberg MR, Lubeck DP, Mehta SS, Carroll PR. Time trends in clinical risk stratification for prostate cancer: implications for outcomes (data from CaPSURE). J Urol 2003; 170 : S21-25.

2. Yossepowitch O, Eggener SE, Bianco FJ JR, CarVER BS, Serio A, ScARDino PT et al. Radical prostatectomy for clinically localized, high risk prostate cancer: critical analysis of risk assesment methods. J Urol 2007; 178: 493-499.

3. Nguyen CT, Reuther AM, Stephenson AJ, Klein EA, JONES JS. The specific definition of high risk prostate cancer has minimal impact on biochemical relapse- free survival. J Urol 2009; 181: 75-80.

4. D’Amico AV, Whittington R, Malkowicz SB, Schultz D, Blank K, Broderick GA et al. Biochemical outcome after radical prostatectomy, external beam radiation therapy, or intersticial radiation therapy for clinically localized prostate cancer. JAMA 1998; 280: 969-974.
5. Bill-Axelson A, Holmberg L, Ruutu M, Häggman M, ANDERSSON SO, BRATELl S et al. Radical prostatectomy versus watchful waiting in early prostate cancer. N Engl J Med 2005: 352: 1977-1984.

6. Zudaire Bergera JJ, Sánchez Zalabardo D, Arocena García-Tapia J, Sanz Pérez G, Díez Caballero F, LóPEz FERRANDIS J et al. Expresión de p53 y Ki67 en piezas de prostatectomía radical. Relación con los datos clínico-patológicos y supervivencia. Actas Urol Esp 2000; 24:307-313.

7. TRUE LD. Surgical pathology examination for the prostate gland: practice survey by the American Society of Clinical Pathologist. Am J Clin Pathol 1994;102:572-579.

8. Amling CL, Bergstralh EJ, Blute ML, SlezaK JM, ZINCKE H. Defining prostate specific antigen progression after radical prostatectomy: what is the most appropiate cut point? J Urol 2001;165:1146-1151.

9. Boorjian SA, Karnes RJ, Rangel LJ, Bergstralh EJ, Blute ML. Mayo Clinic validation of the D'Amico risk group classification for predicting survival following radical prostatectomy. J Urol 2008; 179:1354-1360.

10. Kupelian PA, Elshaikh M, Reddy CA, Zippe C, KLEIN EA. Comparison of the efficacy of local therapies for localized prostate cancer in the prostate- specific antigen era: a large singleinstitution experience with radical prostatectomy and external- beam radiotherapy. J Clin Oncol 2002; 20: 3376-3385.

11. Loeb S, Smith ND, Roehl KA, Catalona WJ. Intermediate- term potency, continence, and survival outcomes of radical prostatectomy for clinically highrisk or locally advanced prostate cancer. Urology 2007; 69:1170-1175.

12. Zelefsky MJ, Eastham JA, Cronin AM, FuKs Zvi, Zhang Z, Yamada Y, Vickers A et al. Metastasis after radical prostatectomy or external beamradiotherapy for patients with clinically localized prostate cancer: a comparison of clinical cohorts adjusted for case mix. J Clin Oncol 2010; 28: 1508-1513.

13. Albertsen PC, Hanley JA, Penson DF, Barrows G, Fine J. 13-year outcome following treatment for clinically localized prostate cancer in a population based cohort. J Urol 2007:177: 932-936.

14. Tewari A, Divine G, Chang P, Shemtov MM, MiLOWSKY M, NANUS D et al. Long- term survival in men with high grade prostate cancer: a comparison between conservative treatment, radiation therapy and radical prostatectomy- a propensity scoring approach. J Urol 2007: 177: 911-915. 
15. Meng MV, Elkin EP, Latini DM, Duchane J, CARROLL PR. Treatment of patients with high risk localized prostate cancer: results from cancer of the prostate strategic urological research endeavor(CaPSURE). J Urol 2005; 173 : 1557-1561.

16. GRuBb RL, KibEL AS. High- risk localized prostate cáncer: role of radical prostatectomy. Curr Opin Urol 2010; 20:241-246.

17. Kane CJ, Presti JC Jr, Amling CL, Aronson WJ, Terris MK, Freedland SJ; SEARCH Database Study Group. Changing nature of high risk patients undergoing radical prostatectomy. J Urol 2007;177:113-117.

18. Stephenson AJ, Kattan MW, Eastham JA, Bianco FJ JR, Yossepowitch O, Vickers AJ et al. Prostate cancer- specific mortality after radical prostatectomy for patients treated in the prostate specific antigen era. J Clin Oncol 2009; 27 : 4300-4305.

19. Godoy G, Tareen BU, Lepor H. Site of positive surgical margins influences biochemical recurrence after radical prostatectomy. BJU Int 2009; 104: 1610-1614.

20. Alkhateeb S, Alibhai S, Fleshner N, Finelli A, Jewett M, ZLotTA A et al. Impact of positive surgical margins after radical prostatectomy differs by disease risk group. J Urol 2010; 183:145-150.

21. Rubio-Briones J, Iborra I, Trassierra M, Collado A, Casanova J, Gómez-Ferrer A et al. Progresión metastásica, mortalidad cáncer especifíca y necesidad de tratamientos de segunda línea en pacientes con cáncer de próstata de alto riesgo tratados inicialmente mediante prostatectomía radical. Actas Urol Esp 2010; 34: 610-617.

22. Ojea CA, Mosteiro Cervino MJ, Dominguez FF, Alonso RA, Rodríguez IB, Benavente DJ et al. The usefulness of $\mathrm{Ki} 67$ expression in the biopsy specimens, to predict the biochemical progression of the prostate cancer after radical prostatectomy. Actas Urol Esp 2004; 28: 650-660.

23. Feneley MR, Young MP, Chinyama C, Kirby RS, PARKInson MC. Ki-67 expression in early prostate cancer and associated pathological lesions. J Clin Pathol 1996; 49: 741-748.

24. Nilsson S, Nordgren H, Karlberg L, Harving B, Busch C, Hall T et al. Expresion of estramustine-binding protein (EMBP) and the proliferation associated antigen $\mathrm{Ki}-67$ in prostatic carcinomas. Scand J Urol Nephrol Suppl 1998; 110: 31-37. 